\title{
MiR-888: A newly identified miRNA significantly over-expressed in endometrial cancers
}

\author{
Adriann M. Hovey ${ }^{1}$, Eric J. Devor ${ }^{1}$ and Kimberly K. Leslie ${ }^{1}$ \\ Key Words: miR-888, microRNA, endometrium, endometrial cancer
}

\begin{abstract}
Endometrial cancer is the most common gynecological malignancy and the fourth most common cancer in women. With accumulating evidence, microRNAs have emerged as significant players in the development and progression of cancers. To investigate the role of miRNAs in endometrial cancer, we used quantitative real-time PCR arrays to identify miRNAs dysregulated in a panel of endometrial cancer tissue samples. Out of 667 miRNAs, miR-888 was identified as one of the most highly over-expressed miRNAs, specifically in mixed müllerian tumors, arguably the most aggressive
\end{abstract}

form of endometrial cancer. Furthermore, realtime PCR validations revealed a significant inverse correlation between age onset of cancer and the level of miR-888 expression. Notably, a potential target of miR-888 is the progesterone receptor, whose role in endometrial cancer is well characterized. Together, these data point to miR-888 playing an important functional role in the development of aggressive endometrial tumors. Future research will focus on identifying and validating the targets of miR-888 to elucidate its mechanism of action and support this hypothesis.

${ }^{1}$ Department of Obstetrics and Gynecology, University of lowa, lowa City, IA, USA, 52242

This research supported in part by NIH 2CA99908-7 and Department of Obstetrics and Gynecology Research and Development Fund.

Corresponding author: Adriann Hovey, Department of Obstetrics and Gynecology, University of lowa, 3234 MERF, 200 Hawkins Drive, lowa City, IA, 52242. Telephone(319) 335-8212 adriann-hovey@uiowa.edu 